\title{
Soluble intracellular adhesion molecule-1 secreted by human umbilical cord blood-derived mesenchymal stem cell reduces amyloid- $\beta$ plaques
}

\author{
J-Y Kim ${ }^{1,2}$, DH Kim ${ }^{1}$, JH Kim ${ }^{1}$, D Lee ${ }^{1}$, HB Jeon ${ }^{1}$, S-J Kwon ${ }^{1}$, SM Kim³ ${ }^{3}$, YJ Yoo ${ }^{4}$, EH Lee ${ }^{2}$, SJ Choi ${ }^{1}$, SW Seo ${ }^{5}$, JI Lee ${ }^{6}$, DL Na ${ }^{5}$, \\ YS Yang ${ }^{1}$, W Oh ${ }^{1}$ and JW Chang ${ }^{\star, 1}$
}

Presently, co-culture of human umbilical cord blood mesenchymal stem cells (hUCB-MSCs) with BV2 microglia under amyloid$\beta 42$ (A $\beta 42$ ) exposure induced a reduction of $A \beta 42$ in the medium as well as an overexpression of the $A \beta$-degrading enzyme neprilysin (NEP) in microglia. Cytokine array examinations of co-cultured media revealed elevated release of soluble intracellular adhesion molecule-1 (sICAM-1) from hUCB-MSCs. Administration of human recombinant ICAM-1 in BV2 cells and wild-type mice brains induced NEP expression in time- and dose-dependent manners. In co-culturing with BV2 cells under A 342 exposure, knockdown of ICAM-1 expression on hUCB-MSCs by small interfering RNA (siRNA) abolished the induction of NEP in BV2 cells as well as reduction of added $A \beta 42$ in the co-cultured media. By contrast, siRNA-mediated inhibition of the sICAM-1 receptor, lymphocyte function-associated antigen-1 (LFA-1), on BV2 cells reduced NEP expression by ICAM-1 exposure. When hUCBMSCs were transplanted into the hippocampus of a 10-month-old transgenic mouse model of Alzheimer's disease for 10, 20, or 40 days, NEP expression was increased in the mice brains. Moreover, A $\beta 42$ plaques in the hippocampus and other regions were decreased by active migration of hUCB-MSCs toward A $\beta$ deposits. These data suggest that hUCB-MSC-derived sICAM-1 decreases $A \beta$ plaques by inducing NEP expression in microglia through the sICAM-1/LFA-1 signaling pathway.

Cell Death and Differentiation (2012) 19, 680-691; doi:10.1038/cdd.2011.140; published online 21 October 2011

Alzheimer's disease (AD) is an irreversible neurodegenerative disease that is accompanied by amyloid- $\beta(\mathrm{A} \beta)$ accumulation, tau aggregation, and chronic inflammation. ${ }^{1}$ Clinical trials evaluating new drugs for $A D$ patients have proved disappointing. For example, phase-III clinical trials of Dimebon, a spotlighted candidate drug for $A D$, failed. ${ }^{2}$ Therefore, the limitations of conventional drugs call for a paradigm shift in $A D$ research.

In this light, stem cell therapeutics for incurable diseases is relevant. Stem cells have the ability to self-renew and differentiate into tissues. ${ }^{3}$ Although human umbilical cord blood mesenchymal stem cells (hUCB-MSCs) are allogeneic stem cells, their transplantation does not induce immunological problems in hosts because these cells do not express major histocompatibility complex class-II molecules and T-cell co-stimulatory factors. ${ }^{4-6}$ In addition, collection of hUCB-MSCs is less invasive and they are easily obtained from cord blood after birth.

Recently, many groups have reported that stem cells have regenerative as well as paracrine effects. The paracrine roles of stem cells have been shown in that stem cell-derived soluble factors can modulate apoptosis, differentiation, proliferation, and inflammation in damaged tissue..$^{7-9}$ In particular, our group has shown that galectin-3, which is secreted by hUCB-MSCs, reduces $A \beta$ neurotoxicity in rat primary neurons. ${ }^{10}$ Furthermore, our group reported that transplantation of hUCB-MSCs improved spatial memory deficits and reduced $\mathrm{A} \beta$ deposits in a transgenic mouse model of AD. ${ }^{11,12}$ Although toxic $A \beta$ deposits were reduced significantly in the hUCB-MSC-transplanted mice, the molecular mechanisms are poorly understood.

The present study sought to clarify the mechanisms involved. Transplantation of hUCB-MSCs in AD transgenic mice induced the expression of the $A \beta$-degrading enzyme neprilysin (NEP) in microglia through a paracrine mechanism. Analysis of the proteins secreted by hUCB-MSCs revealed that soluble intracellular adhesion molecule-1 (sICAM-1) is released from hUCB-MSCs, which, in turn, induces NEP in microglia for $\mathrm{A} \beta$ clearance.

\footnotetext{
${ }^{1}$ Biomedical Research Institute, MEDIPOST Co. Ltd., Seoul 137-874, Republic of Korea; ${ }^{2}$ Department of Physiology, College of Medicine, The Catholic University of Korea, Seoul 137-701, Republic of Korea; ${ }^{3}$ Department of Neurosurgery, Seoul St. Mary's Hospital, The Catholic University of Korea, Seoul, Republic of Korea; ${ }^{4}$ School of Life Sciences, Gwangju-Institute of Science and Technology, Gwangju 500-712, Republic of Korea; ${ }^{5}$ Department of Neurology, Samsung Medical Center, Sungkyunkwan University School of Medicine, Seoul, Republic of Korea and ${ }^{6}$ Department of Neurosurgery, Samsung Medical Center, Sungkyunkwan University School of Medicine, Seoul, Republic of Korea

*Corresponding author: JW Chang, Biomedical Research Institute, MEDIPOST Co. Ltd, 1571-17 Secho3-dong, Secho-gu, Seoul 137-874, Republic of Korea.

Tel: + 8223465 6771; Fax: + 822475 1991; E-mail: jwc@medi-post.co.kr

Keywords: hUCB-MSC; amyloid- $\beta$; Alzheimer's disease; intracellular adhesion molecule-1; paracrine and neprilysin

Abbreviations: hUCB-MSC, human umbilical cord blood-derived mesenchymal stem cell; sICAM-1, soluble intracellular adhesion molecule-1; NEP, neprilysin; AD, Alzheimer's disease; siRNA, small interfering RNA; LFA-1, lymphocyte function-associated antigen-1; CSF, cerebrospinal fluid; Erk-1,2, extracellular signal-regulated kinase; JNK, c-Jun N-terminal kinase; RT-PCR, reverse transcription-PCR; MAP2, microtubule-associated protein-2

Received 04.4.11; revised 25.8.11; accepted 12.9.11; Edited by N Bazan; published online 21.10.11
} 
a

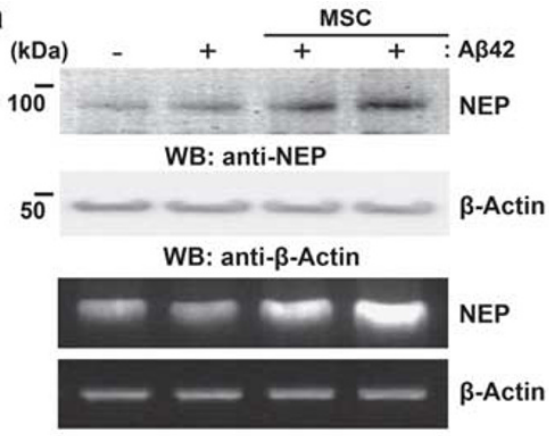

C

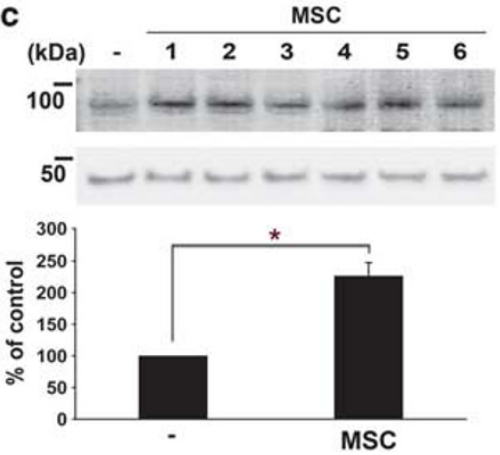

b NEPICD11b
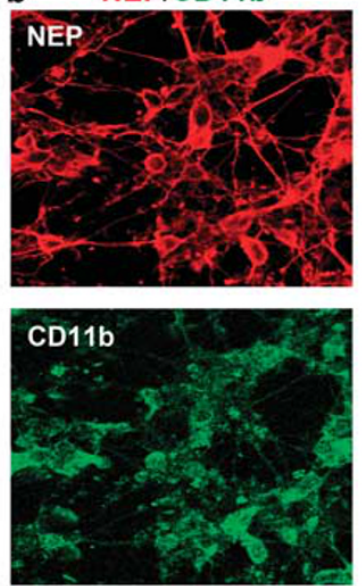

$\beta$-Actin

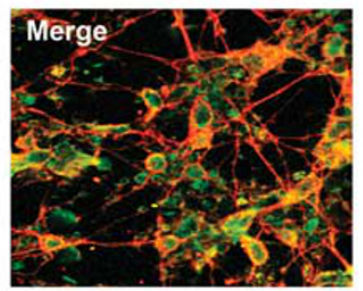

d

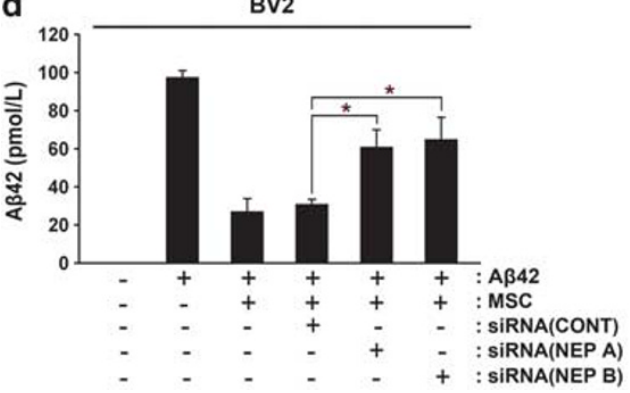

e

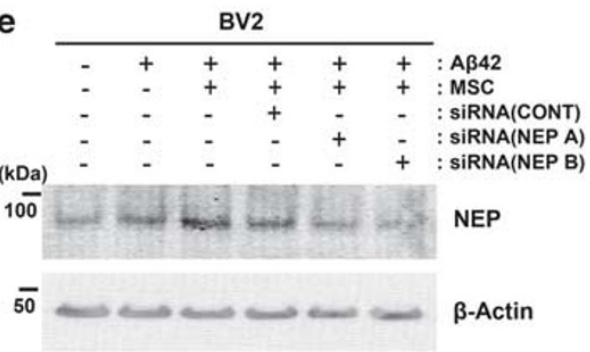

Figure 1 Co-culture with hUCB-MSCs induces NEP expression in microglia. (a) Rat primary neuronal cultures were prepared from 14-day-old embryonic rats as described under Materials and Methods. Rat primary neuronal cultures (in the lower chamber of a Transwell unit) were co-cultured with hUCB-MSCs (in the upper chamber of the Transwell unit) in the presence or absence of $10 \mu \mathrm{M} \mathrm{A} \beta 42$ for $24 \mathrm{~h}$. To examine the levels of mRNA and protein expression of NEP in rat primary cultures, protein extracts or total RNA were analyzed by western blot analysis or RT-PCR, respectively. (b) To examine NEP-expressing cells in rat primary cultures, which contained a mixture of neuronal and glial cells, rat primary cultures co-cultured with hUCB-MSCs were co-stained with antibodies against NEP (red) and the microglia marker CD11b (green). Colocalization of NEP-expressing cells and microglia was detected in a merged image. (c) To examine the reproducibility of induction of NEP expression by co-culturing with hUCB-MSCs, hUCB-MSCs from six different donors were co-cultured with mouse microglial BV2 cells. The levels of NEP expression were analyzed by western blot analysis (densitometric analysis, $n=6$; ${ }^{*} P<0.05$ ). (d) To assess whether the increased NEP of BV2 cells induced by co-culturing with hUCB-MSCs degraded the added A $\beta 42$ (10 $\mu \mathrm{M}$ ) in the cocultured media, the concentration of $A \beta 42$ in each medium was measured by ELISA in the presence and absence of siRNAs for NEP. BV2 cells were pretreated with NEP siRNAs for $14 \mathrm{~h}$ and these cells or only BV2 cells were then co-cultured with hUCB-MSCs in the presence of $\mathrm{A} \beta 42$ for an additional $24 \mathrm{~h}$. The same amount of $\mathrm{A} \beta 42$ ( $10 \mu \mathrm{M}$ ) was added in all conditions. (e) To confirm knockdown of NEP expression in panel $\mathbf{d}$, the above samples were analyzed by western blot analysis using an anti-human NEP antibody. CONT indicates control siRNA

\section{Results}

Co-culture of hUCB-MSCs induces NEP expression in microglia. As transplantation of hUCB-MSCs reduces $\mathrm{A} \beta$ deposits in vivo, ${ }^{11,12}$ we tested whether co-culture with hUCB-MSCs in a Transwell chamber could affect the expression of $\mathrm{A} \beta$ metabolic enzymes, including NEP, in rat primary neuronal cultures. NEP is an $A \beta$-degrading enzyme that is emerging as a therapeutic target in AD. ${ }^{13}$ After coculture with hUCB-MSCs for $24 \mathrm{~h}$ in the presence or absence of $10 \mu \mathrm{M} \mathrm{A} \beta 42, \mathrm{NEP}$ expression in rat primary neuronal cultures was analyzed by reverse transcription-PCR (RT-PCR) and western blot analysis (Figure 1). Co-culture with hUCB-MSCs induced NEP expression in cultured rat primary neuronal culture in the absence and presence of A $\beta 42$ (Figure 1a). As the rat primary neuronal cultures contained a mixture of neuronal and glial cells, we performed a colocalization analysis of NEP-positive cells and microglia (Figure 1b), and neurons (Supplementary Figure S1a). Coculture with hUCB-MSCs in the presence of $\mathrm{A} \beta 42$ demonstrated colocalization of microglia and NEPexpressing cells (red color) by double staining using 
antibodies against CD11b, a marker of microglia (green), and NEP (Figure 1b). Although staining in some microtubuleassociated protein-2 (MAP2)-positive cells (neurons) merged with NEP-expressing cells, the majority of NEP-positive cells were microglia. Moreover, we tested upregulation of NEP in mouse microglial BV2 cells by co-culturing them with hUCBMSCs isolated from six different donors. Western blot analysis revealed that co-culture of six hUCB-MSCs induced an upregulation of NEP in BV2 cells $\left({ }^{*} P<0.05\right.$ versus BV2 cells only; Figure 1c). As co-culture with hUCBMSCs induced NEP expression in BV2 cells, we measured the concentration of added $A \beta 42$ in the co-cultured media to determine whether endogenous NEP was induced in microglia or whether neurons degraded $A \beta 42$ in the media. The level of $A \beta 42$ in the media was compared when hUCBMSCs were co-cultured with BV2 cells or human neuroblastoma SH-SY5Y cells (Supplementary Figure $\mathrm{S} 1 \mathrm{~b}$ ). Significant reductions in $A \beta 42$ concentration were observed in the media of BV2 cells co-cultured with hUCB-MSCs in the presence of $10 \mu \mathrm{M} \mathrm{A} \beta 42$, as compared with the media of $\mathrm{SH}$ SY5Y co-cultured with the same concentration of $A \beta 42$ $\left({ }^{\star} P<0.05\right.$ versus SH-SY5Y alone with $\mathrm{A} \beta 42(n=3$ per group); and ${ }^{\star \star} P<0.05$ versus $\mathrm{BV} 2$ alone with $\mathrm{A} \beta 42(n=3$ per group)). These results may have been due to induction of NEP in the majority of microglia. To determine whether reduction of $A \beta 42$ in the media was mediated by NEP in microglia, two different small interfering RNAs (siRNAs) for NEP were transfected into BV2 cells, which were then cocultured with hUCB-MSCs in the presence of $10 \mu \mathrm{M} \mathrm{A} \beta 42$. The concentration of added $A \beta 42$ was reduced significantly in the media of BV2 cells co-cultured with hUCB-MSCs or control siRNA-treated hUCB-MSCs, as compared with BV2 cells cultured alone in the same amount of $A \beta 42$. By contrast, pretreatment of BV2 cells with two different NEP siRNAs abolished this reduction of $A \beta 42$ in the media upon co-culture with hUCB-MSCs (Figure 1d). The effect of NEP knockdown was evaluated by western blot analysis (Figure 1e). The data indicate that hUCB-MSCs reduced $A \beta 42$ through induction of NEP expression in microglia.

Identification of SICAM-1 as a paracrine factor released by hUCB-MSCs for NEP induction. In Transwell chamber experiments, cells cannot pass through the Transwell membrane because of the restrictive pore size $(1 \mu \mathrm{m})$. Thus, secreted factors released from hUCB-MSCs can act on microglia in the co-culture system. To identify the paracrine factors, cytokine array were performed by using the following conditioned media: hUCB-MSCs or BV2 cells alone; A 342 -treated hUCB-MSCs or BV2 cells; and BV2 cells co-cultured with hUCB-MSCs in the presence or absence of A $\beta 42$ (Figure 2a). Densitometric analysis revealed that interleukin-8 (IL-8), IL-6, growth-regulated oncogene- $\alpha$ $(\mathrm{GRO}-\alpha)$, and ICAM-1 cytokines were markedly increased in the media of BV2 cells co-cultured with hUCB-MSCs in both the presence and in the absence of $A \beta 42$ (Figure $2 b$, $n=3$ per group; ${ }^{\star} P<0.05$ versus MSC alone). Next, we examined which co-cultured cells secreted the identified cytokines. After co-culturing BV2 cells with hUCB-MSCs, either cell type was harvested separately and the expression of each individual cytokine was analyzed by RT-PCR. IL-8,
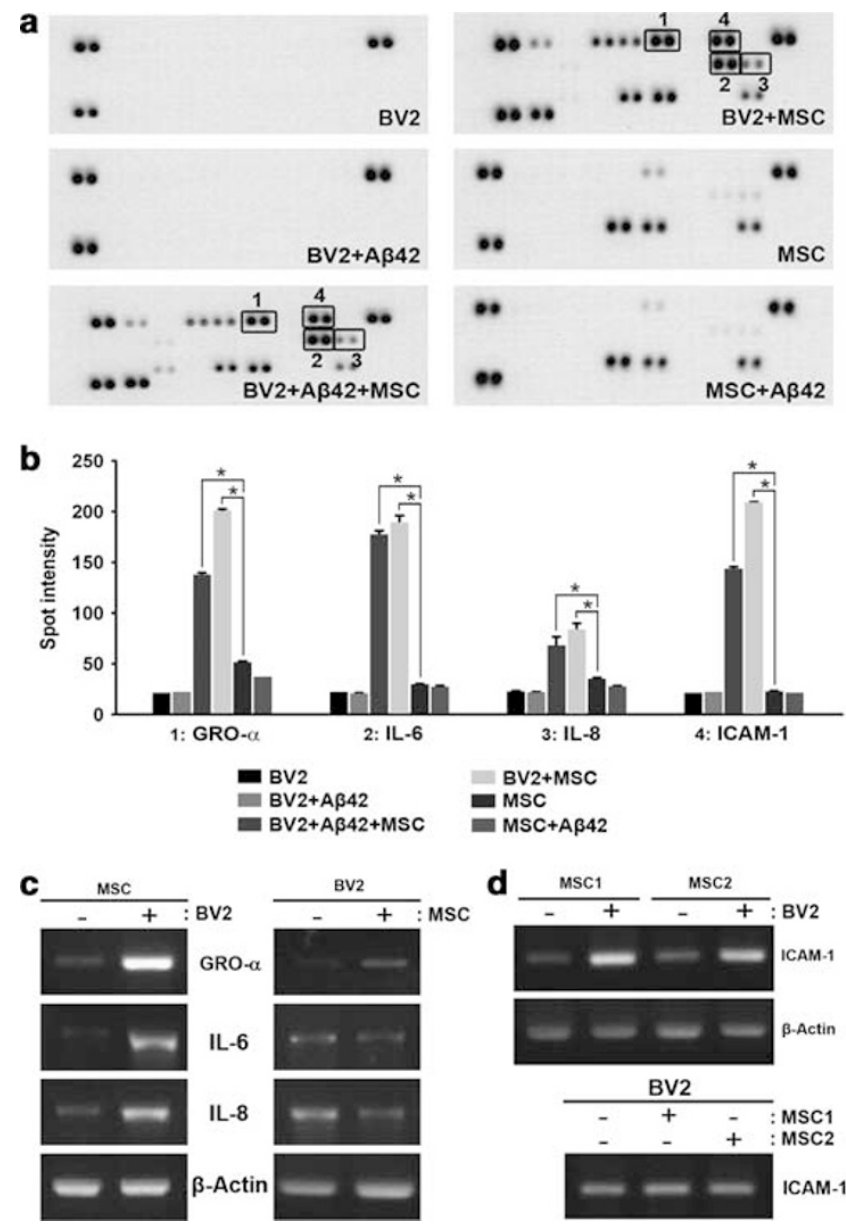

Figure 2 Cytokine analyses identify four proteins that are upregulated in the media of BV2 cells co-cultured with hUCB-MSCs. (a) Medium was collected from BV2 cells and hUCB-MSCs alone; BV2 cells co-cultured with hUCB-MSCs in the presence and absence of $A \beta 42$; and BV2 cells or hUCB-MSCs alone in the presence of $A \beta 42$. Cytokine analysis was performed according to a recommended protocol. The boxed area indicates upregulation of four proteins in the medium from BV2 cells co-cultured with hUCB-MSCs in the presence or absence of A $\beta 42$. (b) A typical result of densitometric analysis obtained from three independent experiments ( $n=3$ per group; ${ }^{*} P<0.05$ versus MSC alone). (c) To determine which cells secreted GRO- $\alpha$, IL-6, IL-8, and ICAM- 1 when co-cultured in the Transwell system, BV2 cells and hUCB-MSCs were co-cultured and then analyzed by RT-PCR using a specific primer for human GRO- $\alpha$, LL-6, and IL-8. (d) In the same experiment as in panel $\mathbf{c}, \mathrm{BV} 2$ cells were co-cultured with two different $\mathrm{hUCB}-\mathrm{MSC}$ to analyze ICAM-1 expression in RT-PCR using a primer for human ICAM-1

IL-6, GRO- $\alpha$, and ICAM-1 were overexpressed in co-cultured hUCB-MSCs but not in BV2 cells (Figures $2 \mathrm{c}$ and d). In particular, ICAM-1 levels were increased by co-culturing BV2 cells with hUCB-MSCs obtained from two individuals (Figure 2d). These data demonstrate that increased levels of ICAM-1 were released from hUCB-MSCs by co-culturing with BV2 cells.

Among these cytokines, only ICAM-1 treatment induced the expression of NEP in BV2 cells in time- and dose-dependent manners (Figure $3 a,{ }^{\star} P<0.05$ versus without recombinant ICAM- $1 ; n=3$ per group). As NEP expression was upregulated by ICAM-1 in the absence and presence of $A \beta 42$ (Figure 3a), a dose of 500 or $1000 \mathrm{ng} / \mathrm{kg}$ of human 

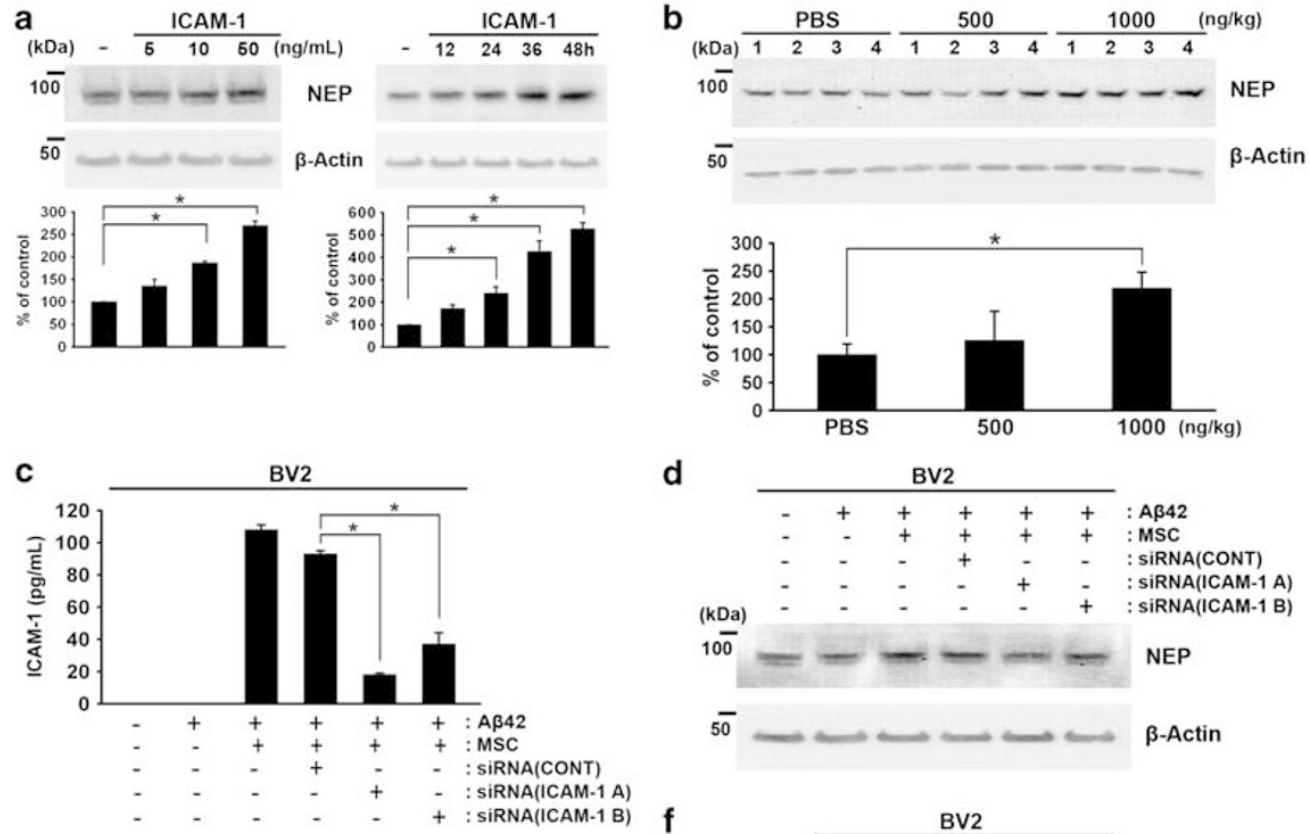

d
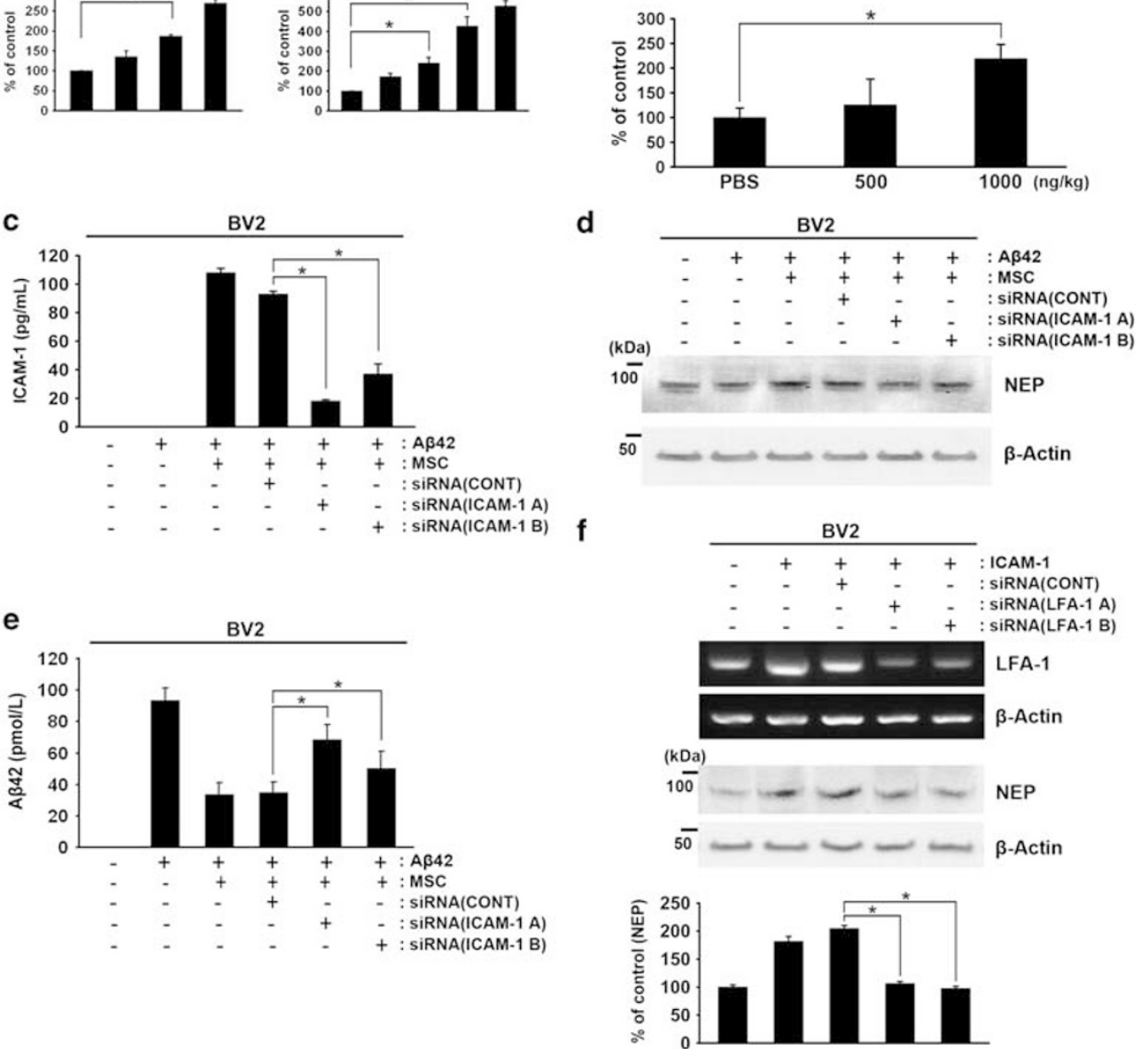

Figure 3 Identification of hUCB-MSC-derived SICAM-1 as an inducer of NEP expression in microglia. (a) BV2 cells were treated with human recombinant ICAM-1 in a dose- $(5,10$, and $50 \mathrm{ng} / \mathrm{ml})$ and time-dependent $(12,24,36$, or $48 \mathrm{~h})$ manner. Each cell lysate was analyzed with an anti-NEP antibody by western blot analyses. Densitometric analyses indicated an increase of NEP in three independent experiments $\left({ }^{*} P<0.05\right)$. (b) Dose-dependent human recombinant ICAM-1 $(500$ and $1000 \mathrm{ng} / \mathrm{kg})$ was injected bilaterally into the hippocampus of $\mathrm{B} 6 \mathrm{C} 3$ mice for 7 days. As control, PBS was injected into both hippocampi. The collected brains were analyzed by western blot analysis using an anti-NEP antibody. NEP levels were upregulated at $1000 \mathrm{ng} / \mathrm{kg}$ when analyzed by densitometric analysis $\left(n=4\right.$ per group; ${ }^{*} P<0.05$ versus PBS control). (c) To knock down ICAM-1 in hUCB-MSCs, hUCB-MSCs were pretreated with an siRNA control (siCONT) or siRNA (A) and siRNA (B) of ICAM-1 for $14 \mathrm{~h}$, and then these cells were cocultured with BV2 cells for an additional $24 \mathrm{~h}$ in the presence of $\mathrm{A} \beta 42$. The levels of sICAM- 1 in each media were measured by ELISA ( ${ }^{*} P<0.05 ; n=3$ per group). (d) BV2 cells were harvested from the above conditions and analyzed by western blot analysis using an anti-NEP antibody. (e) To determine whether the reduced sICAM-1 secretion affected the ability of $A \beta 42$ reduction by hUCB-MSCs, the A $\beta 42$ levels in the media were measured by A $\beta 42$ ELISA. (f) To knock down LFA-1, BV2 cells were pretreated for $14 \mathrm{~h}$ with two different siRNAs for LFA-1, siRNA LFA-1 A and LFA-1 B, and then these cells were exposed to recombinant ICAM-1 (20 ng/ml) for $24 \mathrm{~h}$. Knockdown of LFA-1 was confirmed by RT-PCR. Reduced NEP expression by siRNAs of LFA-1 was confirmed by western blot analysis using an anti-NEP antibody. Densitometric analysis of NEP was performed in three independent experiments $\left({ }^{*} P<0.05 ; n=3\right.$ versus siCONT-treated BV2 with ICAM-1)

recombinant ICAM-1 or phosphate-buffered saline (PBS) was administered to the hippocampus of 10-month-old littermate mice for 7 days. After 7 days, the brain tissue was analyzed by western blot analysis to determine the levels of NEP expression. NEP levels were upregulated in the $1000 \mathrm{ng} / \mathrm{kg}$ ICAM-1-injected groups (Figure $3 \mathrm{~b},{ }^{\star} P<0.05$ versus PBS control; $n=4$ each group). In addition, pretreatment of hUCBMSCs with two different ICAM-1 siRNAs reduced the levels of secreted sICAM-1, as well as overexpression of NEP, in BV2 cells co-cultured with hUCB-MSCs (Figures $3 \mathrm{c}$ and $\mathrm{d}$ ). To examine knockdown of sICAM-1 in hUCB-MSCs, medium was collected and ICAM-1 levels were analyzed by ELISA (Figure 3c, ${ }^{\star} P<0.05$ versus the control siRNA-treated group; $n=3$ per group). Consequently, knockdown of ICAM-1 in hUCB-MSCs reduced the degradation of $A \beta 42$ (Figure $3 e$ ), as well as NEP expression (Figure $3 d$ ), in the BV2 cells in the co-culture system $\left({ }^{\star} P<0.05\right.$ versus the control siRNA-treated group; $n=3$ per group). These data indicate that SICAM-1 derived from hUCBMSCs induced the expression of NEP in microglia and a reduction in $A \beta 42$ levels. 
To investigate the signal pathway of ICAM-1-induced NEP expression on microglia, we tested whether ICAM-1 could interact with its known receptor. As lymphocyte functionassociated antigen-1 (LFA-1), integrin- $\beta 2$ has been proposed as the ICAM-1 receptor in lymphocytes, ${ }^{14,15}$ we tested whether knockdown of LFA-1 expression in BV2 cells by two different specific siRNAs of LFA-1 could abolish NEP expression by ICAM-1 treatment. When LFA-1 expression was reduced by these siRNAs, ICAM-1-induced NEP expression was also significantly downregulated (Figure 3f). Knockdown of LFA-1 on BV2 was confirmed by RT-PCR and immunostaining (Supplementary Figure S2). These data indicated that ICAM-1-induced NEP expression could be regulated by the integrin- $\beta 2$ LFA-1-mediated signaling pathway.

Knockdown of ICAM-1 reduces the neuroprotective effects of hUCB-MSCs against A/42 neurotoxicity. To determine the role of SICAM-1 in the neuroprotective effects of hUCB-MSCs in $\mathrm{A} \beta 42$-exposed rat primary neuronal cultures, we compared the neuroprotective effects of hUCB-MSCs in the absence and presence of two different ICAM-1 siRNAs. Reduced levels of SICAM-1 in the media were confirmed by ELISA (Figure 4a). Reduction of $A \beta 42$ concentration was observed in rat primary neuronal cultures after co-culturing with control siRNA-treated hUCB-MSCs, as compared with co-culture with ICAM-1 siRNA-treated hUCB-MSCs (Figure 4b) ( ${ }^{\star} P<0.05$ versus siRNA control treated hUCB-MSC; $n=3$ per group). Although hUCB-MSCs alone protected $\mathrm{A} / 442$-exposed, MAP2-positive rat primary neurons, ICAM-1 siRNA-treated hUCB-MSCs did not protect neurons from death (Figures $4 \mathrm{c}$ and $\mathrm{d}$ ). The percentage of surviving neurons was analyzed by counting the number of MAP2-positive cells in each condition ( $n=3$ per group; $\left.{ }^{\star} P<0.05\right)$. These data indicate that the neuroprotective effects of hUCB-MSCs were mediated by the NEP expression induced by SICAM-1.

Transplantation of hUCB-MSCs induces NEP expression in vivo. To evaluate the induction of NEP expression by hUCB-MSCs in vivo, we performed bilateral transplantation of hUCB-MSCs $\left(2 \times 10^{4}\right.$ cells per head $)$ into the hippocampal tissues of 10-month-old-APP/PS1 double-transgenic mice in a time-dependent manner. As control, human skin fibroblast Hs68 cells were administered (Figure 5a). To check whether injection was performed in accurate positions in the hippocampus, brain tissues at 10 days were stained by a human-specific anti- $\beta 2$-microglobulin (brown). The majority of transplanted hUCB-MSCs were stained in the needle track of the hippocampus at 10 days (Figure $5 \mathrm{~b}$ ). Although these antibodies detected human chondrocytes, they did not detect
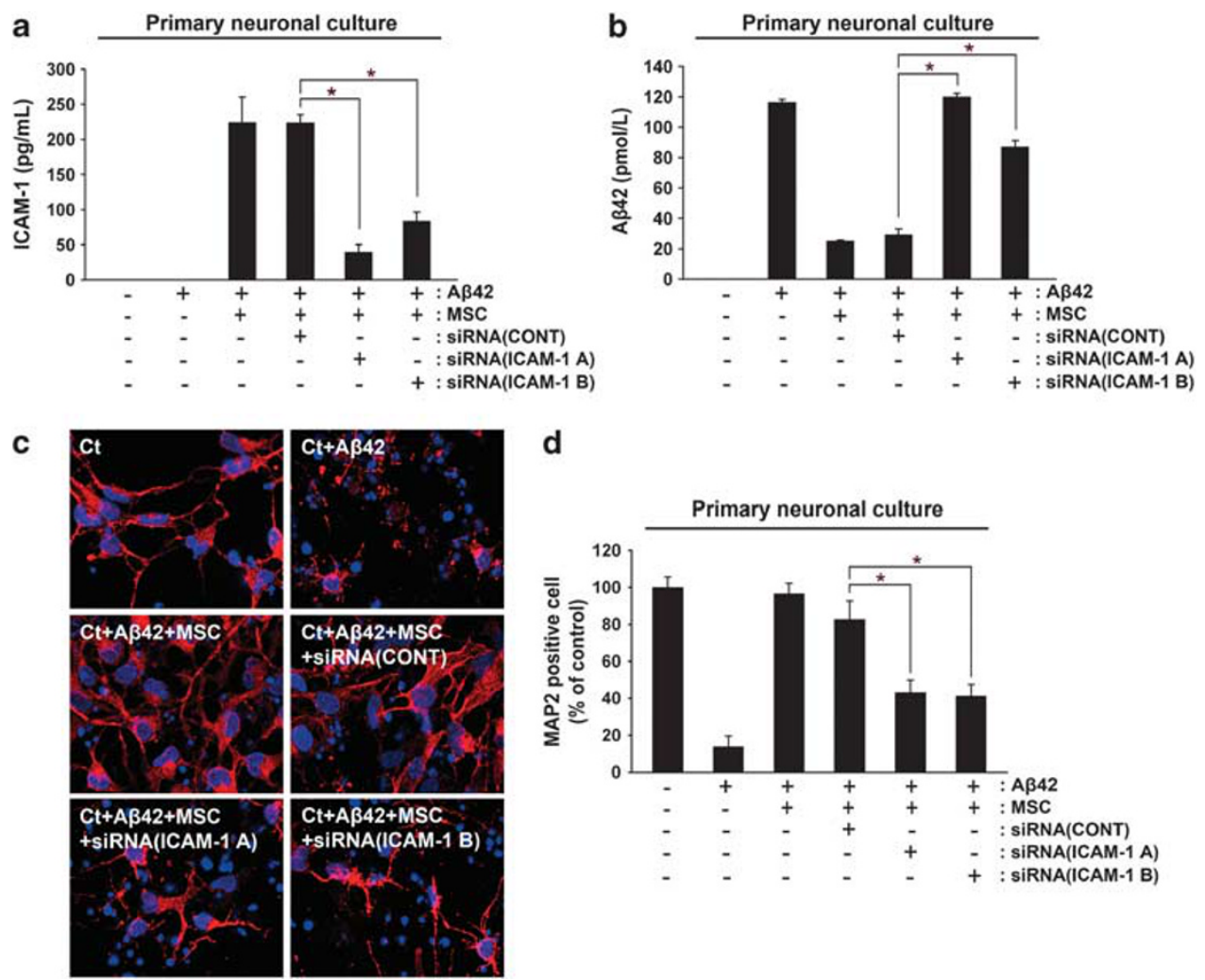

Figure 4 Knockdown of ICAM-1 reduces the neuroprotective effects of hUCB-MSCs against A $\beta 42$ neurotoxicity. (a and $\mathbf{b}$ ) As co-culturing with hUCB-MSCs protects rat primary neuronal cell cultures from A $\beta 42$ neurotoxicity, ${ }^{10}$ we evaluated the effects of ICAM- 1 knockdown in hUCB-MSCs. After hUCB-MSCs were pretreated with siRNA (A) and siRNA (B) for $14 \mathrm{~h}$, these hUCB-MSCs were co-cultured with rat primary neuronal cells. The levels of sICAM-1 and A $\beta 42$ were each confirmed by ELISA. (c) Rat primary neuronal cells in the lower chamber were then stained with an anti-MAP2 antibody in order to count the number of surviving neurons. (d) Neuroprotection of hUCB-MSCs was calculated by the percentage of the number of MAP2-positive neurons per number of DAPI-stained cells. Ct indicates cortical neuron. ${ }^{*} P<0.05$ versus control siRNA treated MSC with $\mathrm{A} \beta 42$ 


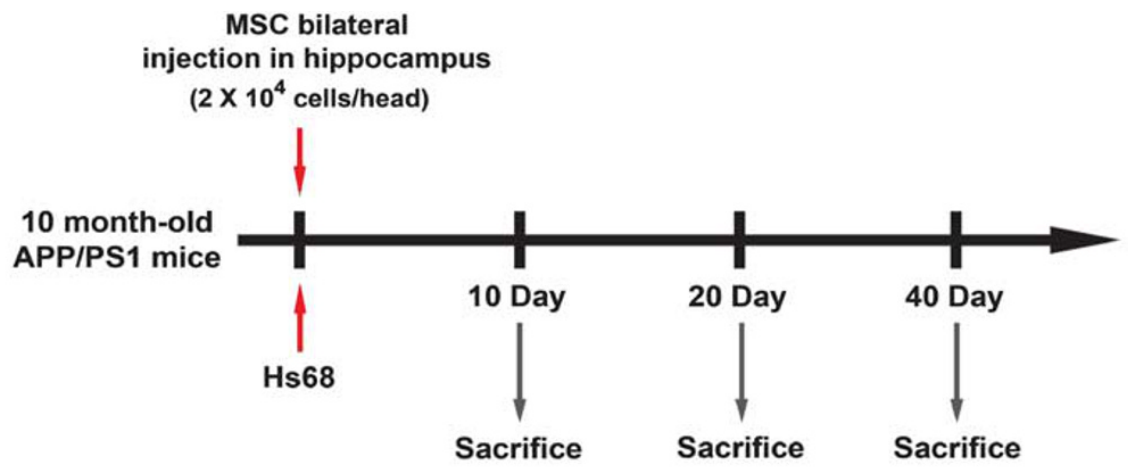

b

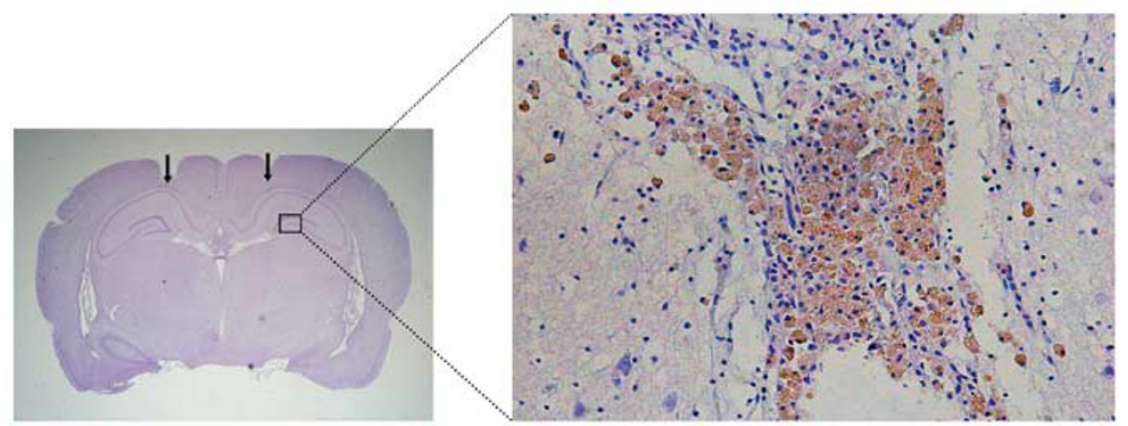

C

MSC
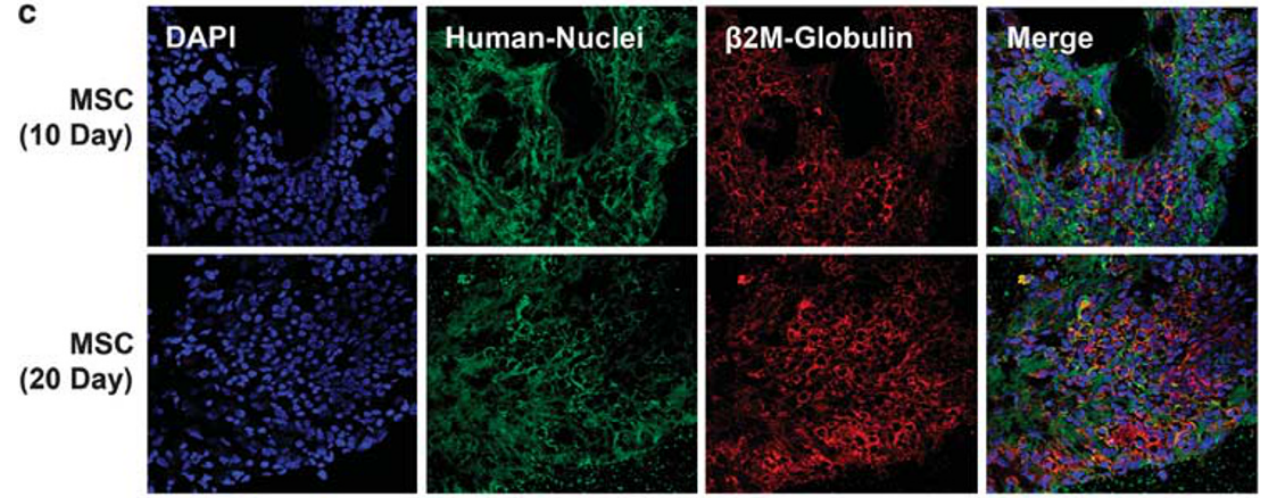

MSC
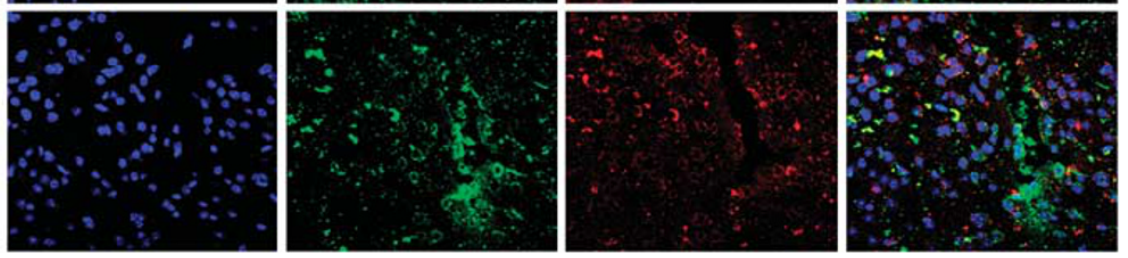

Figure 5 Transplantation of hUCB-MSCs in the brain parenchyma of APP/PS1 transgenic mice. (a) hUCB-MSCs $\left(2 \times 10^{4}\right.$ cells per head) or human skin fibroblasts, Hs68 $\left(2 \times 10^{4}\right.$ cells per head), were administered bilaterally into the hippocampus of 10-month-old APP/PS1 double-transgenic mice. Mice were Killed at 10, 20, and 40 days after cell administration. (b) To detect transplanted hUCB-MSCs in mice brain, coronal sections of mice brain were co-stained using human-specific anti- $\beta 2$-microglobulin and hematoxylin. The magnified box area indicates $\beta 2$-microglobulin-positive hUCB-MSCs in the needle track. (c) To analyze the duration of survival of hUCB-MSCs in the brain, each brain tissue at 10, 20, and 40 days was co-stained by using the two human-specific antibodies, anti- $\beta 2$-microglobulin (red) and anti-human nuclei (green)

$\beta 2$-microglobulin in mouse liver and muscle (Supplementary Figure S3). To analyze the survival of transplanted hUCBMSCs, we double stained using an antibody for humanspecific anti-human nuclei (green) and human $\beta 2$-microglobulin (red) at 10, 20, and 40 days. The stained hUCBMSCs were clearly detected, and the red and blue cells were completely merged in images collected at 10 and 20 days.
However, hUCB-MSCs were significantly reduced at 40 days (Figure $5 c$ ) in all brain areas. Before transplantation of the hUCB-MSCs, we examined the levels of NEP expression in the transgenic mice at various ages. NEP expression was significantly reduced at 12 and 18 months (Figure 6a, ${ }^{\star} P<0.05$ versus 6 -month-old transgenic mice; $n=4$ per group). However, A $\beta 42$ accumulation was noted. After 
a

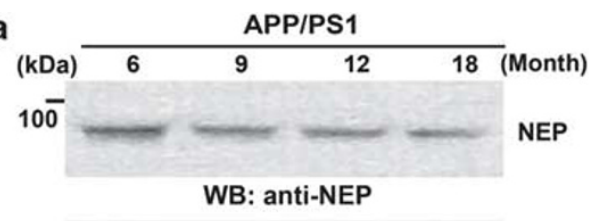

10

WB: anti-NEP

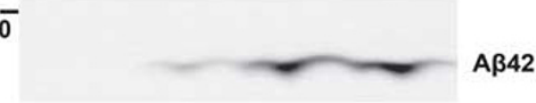

WB: anti-A $\beta 42$

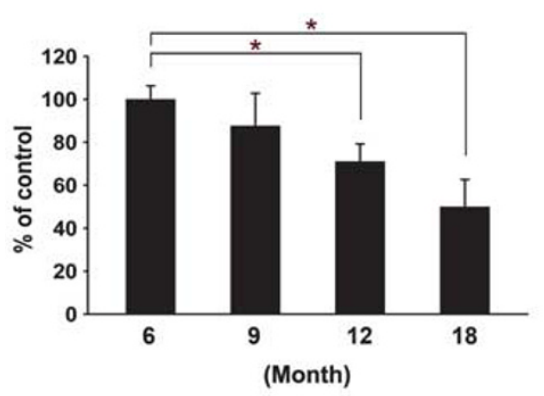

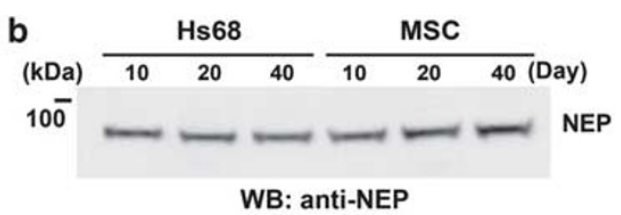

$15 \overline{0}$

WB: anti-NEP

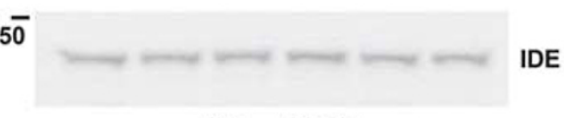

WB: anti-IDE

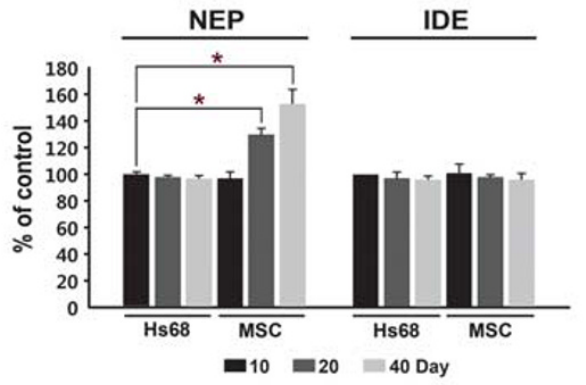

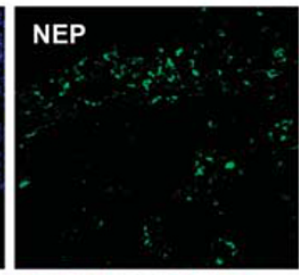
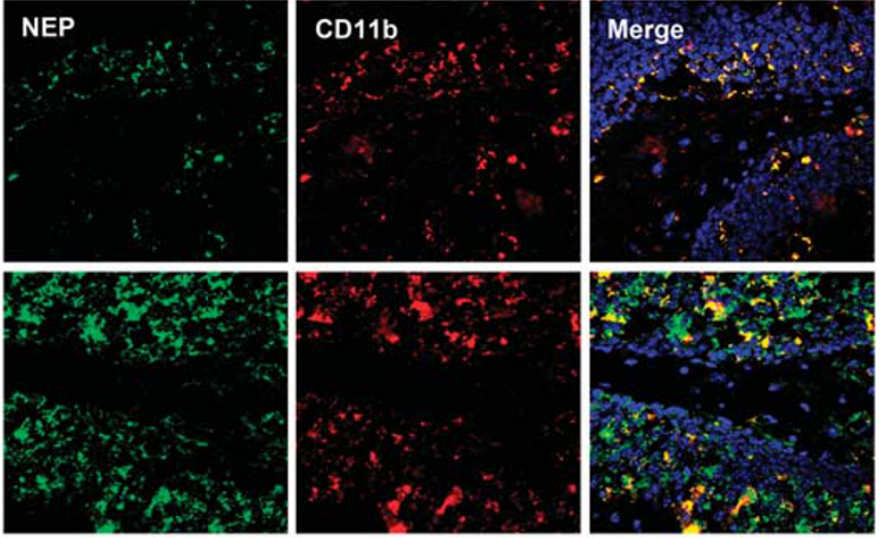

\section{Microglia}

Figure 6 Transplantation of hUCB-MSCs induces NEP expression in microglia in APP/PS1 mice. (a) Densitometric analyses $\left(n=4\right.$; $\left.{ }^{*} P<0.05\right)$ of western blot analyses were performed to determine the levels of NEP expression and A $\beta 42$ accumulation in APP/PS1 mice at different ages (6, 9, 12, and 18 months). (b) After transplantation of Hs68 and hUCB-MSCs $\left(2 \times 10^{4}\right.$ cells per head) at 10, 20, and 40 days in 10-month-old APP/PS1 mice, each brain tissue (not including the cerebellum) was analyzed by western blot analysis using an anti-NEP antibody and an anti-IDE antibody. The band volume of NEP was analyzed by densitometry $\left(n=4\right.$; $\left.{ }^{*} P<0.05\right)$. (c) Coronal brain sections from animals at 20 days were double-stained using CD11b and NEP antibodies. The hippocampus region was examined by confocal microscopy. The red/green color shows CD11b-positive/NEP-positive cells. Two images were combined into a merged image

transplantation of hUCB-MSCs and Hs68, mice were killed at 10,20 , and 40 days to perform western blot analyses and immunohistochemistry (IHC). As in our in vitro data, NEP expression was increased in a time-dependent manner by transplantation of hUCB-MSCs as compared with the Hs68injected group (Figure $6 \mathrm{~b}$ ). In densitometric analysis, NEP expression was significantly increased by an average of $20 \%$ and $38 \%$ at 20 and 40 days, respectively, after hUCB-MSC transplantation, as compared with 10 days in the Hs68 controls (Figure 6b, ${ }^{*} P<0.05 ; n=4$ per group). Another $\mathrm{A} \beta$ degrading enzyme, insulin-degrading enzyme (IDE), ${ }^{16}$ was also applied to the same tissue. IDE expression levels were not changed by hUCB-MSCs (Figure 6b).

To analyze the colocalization of microglia and NEP expression in the hippocampal region of 20-day-old mice, double staining was performed using antibodies against NEP and CD11b in coronal brain sections (Figure 6c). It was difficult to observe any colocalization of microglia and NEP expression in the Hs68injected group $(n=4)$. However, NEP expression was clearly noted in the microglia of the group transplanted with hUCBMSCs $(n=4)$. Colocalization of neurons and NEP expression was also examined in tissues. A few MAP2-positive neurons colocalized with NEP-expressing cells, as compared with CD11b-positive microglia (Supplementary Figure S4). These data indicate that transplanted hUCB-MSCs induced NEP expression in the microglia of AD-transgenic mice.

Transplantation of hUCB-MSCs reduces $A \boldsymbol{\beta}$ plaques in vivo. To confirm whether the number of $A \beta$ plaques was affected by transplantation of hUCB-MSCs, thioflavin-S 
staining of $\mathrm{A} \beta$ plaques was performed in each of the tested tissues from 40-day-old mice, although the majority of hUCB-MSCs had disappeared (Figure 7A). The boxed area in the images was magnified to visualize $\mathrm{A} \beta$ plaques in each coronal section. $\mathrm{A} \beta$ plaques in the hippocampal ( $a$ and $b$ ) and cortical regions ( $c$ and $d$ ) from Hs68-injected and hUCB-MSC-injected groups (Figure 7A) were magnified. Quantitative image analysis of the area occupied by the
$\mathrm{A} \beta$ plaques showed statistically significant reductions (Figure $7 \mathrm{~B},{ }^{\star} P<0.05 ; n=4$ per group) in hUCB-MSCtransplanted mice, as compared with Hs68-injected APP/PS1 mice. Additionally, reduced A $\beta 42$ monomers were also confirmed by western blot analysis using an anti-A $\beta 42$ antibody (Figure $7 \mathrm{C},{ }^{*} P<0.05$ versus the Hs68-injected group; $n=4$ per group). Furthermore, ELISA analyses of the same brain extracts revealed
A
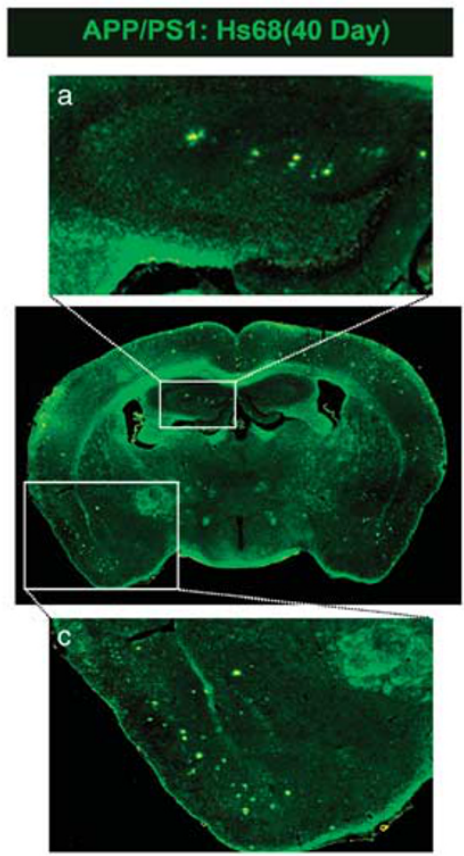

B

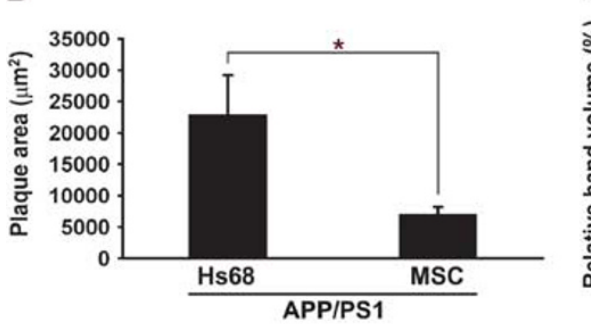

D
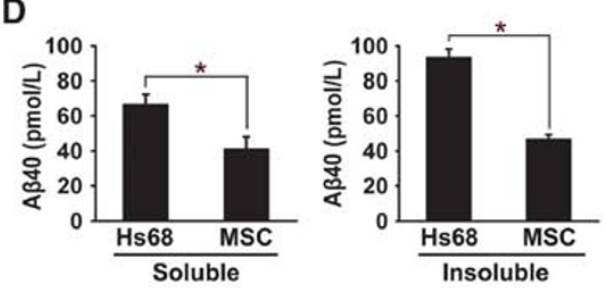

C
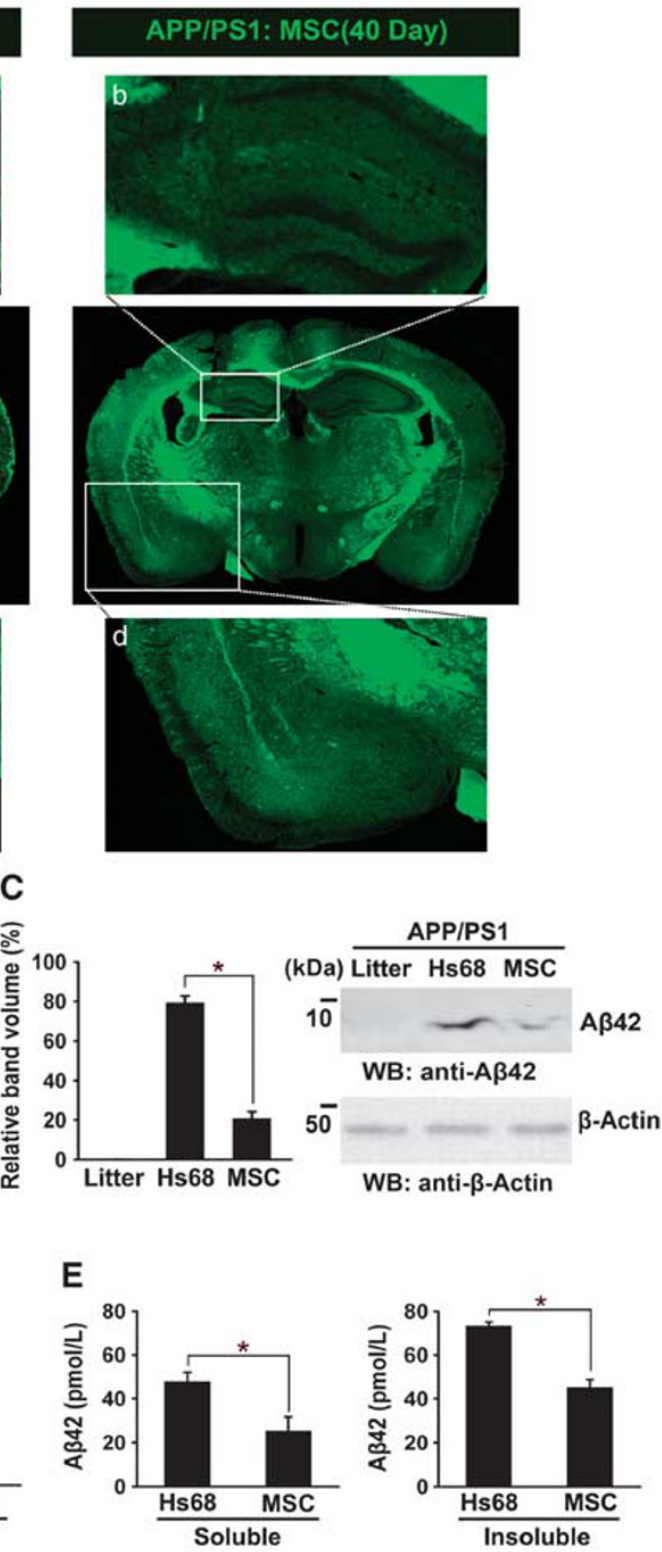

Figure 7 Transplantation of hUCB-MSCs reduces A $\beta$ plaques in APP/PS1 mice. (A) Brain sections from the Hs68 and hUCB-MSC-injected groups that survived for 40 days were stained with thioflavin-S to visualize $A \beta$ plaques. The yellowish dots indicate $A \beta$ plaques. Images $a$ and $b$ indicate the enlarged hippocampal region, and images $C$ and $d$ show the magnified cortical region. (B) A $\beta$ plaques were measured by image analysis ( $n=4$ per group; ${ }^{\star} P<0.05$ versus the Hs68-injected group). (C) The levels of A $\beta 42$ were detected in brain tissues, including the littermate, the Hs68- and hUCB-MSC-injected groups, by western blot analysis using an anti-A $\beta 42$ antibody. Litter indicates littermate of APP-PS1 mice. Densitometric analysis revealed decreased A $\beta 42$ level in the hUCB-MSC-injected group ( ${ }^{*} P<0.05$ versus the Hs68-injected group; $n=4$ ). Detergent-soluble and insoluble A $\beta 40$ (D) or A $\beta 42$ (E) were analyzed by specific ELISA ( ${ }^{*} P<0.05$ versus the Hs69-injected group; $n=4$ per group). Preparation of insoluble and soluble $A \beta$ was performed as described by Nikolic et $a .^{29}$ 
that the levels of both detergent-soluble and -insoluble $A \beta 40$ and $A \beta 42$ were reduced significantly in the hUCB-MSC injected groups (Figures $7 \mathrm{D}$ and $\mathrm{E}$, ${ }^{*} P<0.05 ; n=4$ per group). As the results of thioflavin-S staining correlated with western blot analysis using anti-A $\beta 42$ antibody and ELISA, these data indicate that hUCB-MSC transplantation decreased $\mathrm{A} \beta$ plaques through NEP expression of microglia.

Detection of hUCB-MSCs expressing ICAM-1 in other brain areas. Although hUCB-MSCs were transplanted only in the hippocampus, $A \beta$ plaques in remote cortices from the hippocampus were reduced markedly (Figure 7 ). From this phenomenon, we tested whether hUCB-MSCs could migrate to other brain areas such as the cortex in the frontal lobe. Brain tissues were collected from the frontal lobe of hUCBMSC-transplanted $A D$ mice at 20 days and analyzed by using anti-human nuclei (green) and ICAM-1 (red) antibodies to analyze the colocalization of ICAM-1 and hUCB-MSC. Interestingly, hUCB-MSCs expressing ICAM-1 were detected in other brain areas at 20 days in $A D$ mice (Figures 8A-E). hUCB-MSCs expressing ICAM-1 (orange cells in the merged image) were detected in the neocortex, hypothalamus, amygdale, and striatum. Each region $(a-e)$ is marked in Figure 8F. In our previous reports, ${ }^{17-19}$ hUCBMSC migrated toward the cell secreting the inflammatory cytokine IL-8 in vitro and in vivo. Microglia that secrete inflammatory cytokines are usually localized near $\mathrm{A} \beta$ plaques in $A D .^{20-22}$ Based on these findings, migrating hUCB-MSCs (green) were often observed near $A \beta$ deposits (red) in brain tissues (Figure $8 \mathrm{G}$ ). This migration toward $\mathrm{A} \beta$ plaques was too robust, because hUCB-MSCs injected into the cerebrospinal fluid (CSF) through the cisterna magna also migrated to the brain parenchyma. For example, under the subarchoid space (yellow dashed line), part of the circulation route of the CSF, migrating hUCB-MSCs were detected near $\mathrm{A} \beta$ deposits on the edge of cortex (box $\mathrm{h}$ in Figure 8F) (Figure $8 \mathrm{H}$ ). Surprisingly, when hUCB-MSCs were inoculated in the hippocampus or the CSF in normal mice, hUCB-MSC migration was not found in other brain areas (data not shown). These data indicated that $A \beta$ plaques located remotely from injection sites could be removed by migration of hUCB-MSCs toward $\mathrm{A} \beta$ deposits.

\section{Discussion}

In stem cell therapy, several trials using AD models have been conducted by using transplantation of rat or mouse neural stem cells. ${ }^{23-25}$ In these trials, $\mathrm{A} \beta$, IDE, and the tau status were not altered by transplantation of neural stem cells. The majority of these studies have demonstrated that transplantation of neural stem cells ameliorates AD-associated behavioral deficits. Here, for the first time, we demonstrate removal of $\mathrm{A} \beta$ through hUCB-MSC-derived SICAM-1 by induction of endogenous NEP in a mouse model of AD.

The metabolism of $\mathrm{A} \beta$ is a major target for new drug development. In particular, NEP is an emerging target for $\mathrm{A} \beta$-targeted drugs, because decreased $\mathrm{A} \beta$ clearance in $\mathrm{AD}$ patients was reported. ${ }^{26}$ Therefore, recovery of NEP expression in patients with $A D$ may be a possible strategy for $A D$ therapy.

In this study, expression of the $A \beta$ clearance enzyme, NEP, was induced by co-culturing hUCB-MSCs in microglia cells. NEP induced in the media was able to degrade $A \beta 42$ because knockdown of NEP in BV cells abolished $A \beta$ removal by hUCB-MSC co-culture (Figure 1). Moreover, transplantation of hUCB-MSCs at 10,20, and 40 days in the bilateral hippocampus also induced a time-dependent NEP expression in AD-transgenic mice (Figure 6). These data support the idea that reduction of $\mathrm{A} \beta$ plaques by hUCB-MSCs is due to induction of NEP in mouse brain. In this study, we examined how hUCB-MSCs can induce NEP in microglia by using a Transwell system. From cytokine analyses of the conditioned media, we identified release of ICAM-1 by hUCB-MSCs (Figure 2) and found that recombinant ICAM-1 induced NEP expression in vitro and in vivo (Figure 3). In addition, knockdown of ICAM-1 expression in hUCB-MSCs abolished the reduction of $\mathrm{A} \beta 42$ and NEP expression in BV2 cells (Figure 3). Consequently, the neuroprotective effects of hUCB-MSCs were inhibited significantly in rat primary cultures with inhibition of ICAM-1 expression in hUCB-MSCs (Figure 4). These data are consistent with the view that sICAM-1 from hUCB-MSCs induces the expression of NEP in microglia, which then remove $A \beta$ plaques (Figure 9).

ICAM-1 is a cell-surface molecule that is expressed by multiple types of cells, including epithelial cells, leukocytes, endothelial cells, and fibroblasts. It is a member of the immunoglobulin superfamily of adhesion molecules that are involved in cell-cell and cell-matrix interactions. Although the predominant function of ICAM-1 is cell adhesion, soluble and circulating forms of ICAM-1 are detected in body fluids, including serum and the CSF. ${ }^{27}$ Presently, ICAM-1 expression in naïve hUCB-MSCs was low because expression of ICAM-1 mRNA or SICAM-1 in media was detected only in the media of hUCB-MSCs co-cultured with BV2 cells (Figure 2). LFA-1 is a receptor for ICAM-1 in lymphocytes. ${ }^{14,15} \mathrm{We}$ confirmed that knockdown of LFA-1 expression in BV2 cells abolished ICAM-1-induced NEP expression in these cells (Figure 3f). Especially, ICAM-1 stimulates downstream extracellular signal-regulated kinase (Erk)-1,2 activation in T-cell receptor activation through the LFA-1 signaling pathway. ${ }^{28}$ In this study, we examined several major kinases during ICAM-1 exposure in BV2 cells. Among these, c-Jun $\mathrm{N}$-terminal kinase (JNK) and Erk-1,2 were activated in a timedependent manner. Inhibition of these two kinases by specific inhibitors did not affect NEP expression by ICAM-1 in BV2 cells (data not shown). Although LFA-1/ICAM-1 interacts in BV2 cells, such as occurs in lymphocytes, the downstream signaling pathway should be investigated using phosphokinase arrays to find novel pathways. In conclusion, NEP induction in microglia by ICAM-1 must be mediated by the LFA-1, integrin- $\beta 2$ pathway.

In this study, we observed an additional role of hUCB-MSCderived sICAM-1 in microglial function. In a previous report, Nikolic et al. ${ }^{29}$ reported that administration of hUCB-mononuclear cells (MNCs) decreases $\mathrm{A} \beta$ plaques by disrupting CD40/CD40L activity in microglia. Interestingly, Tan et al. reported that CD40 ligation promotes proinflammatory activation of microglia and reduces microglial phagocytosis of 


\section{A}

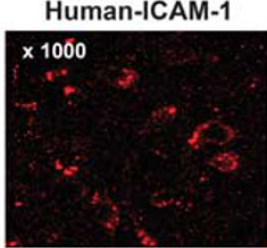

B

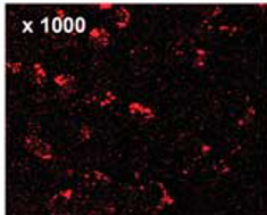

C

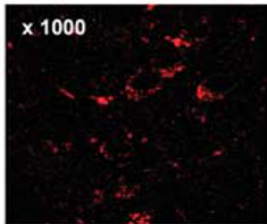

D

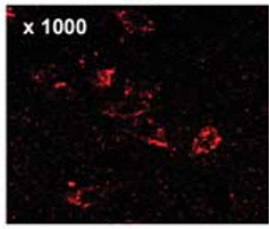

E

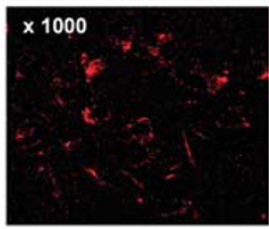

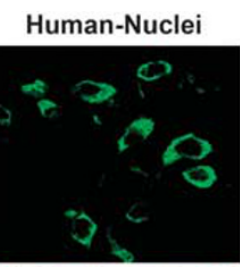
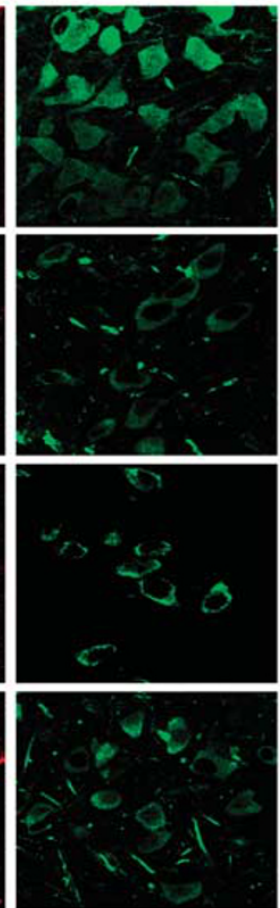
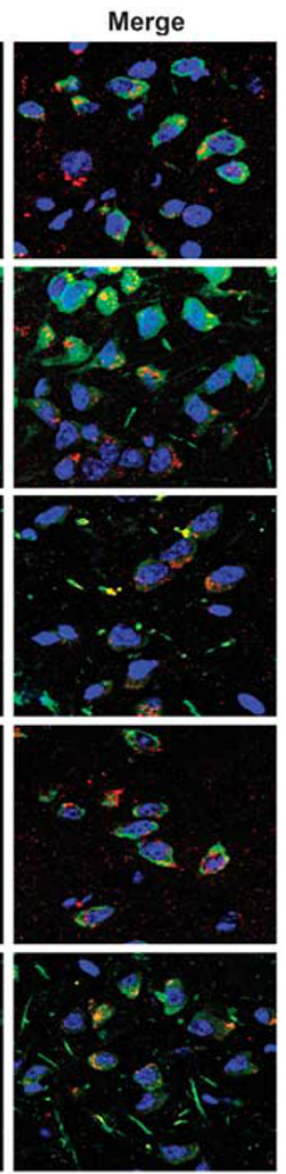

\section{$\mathbf{F}$}
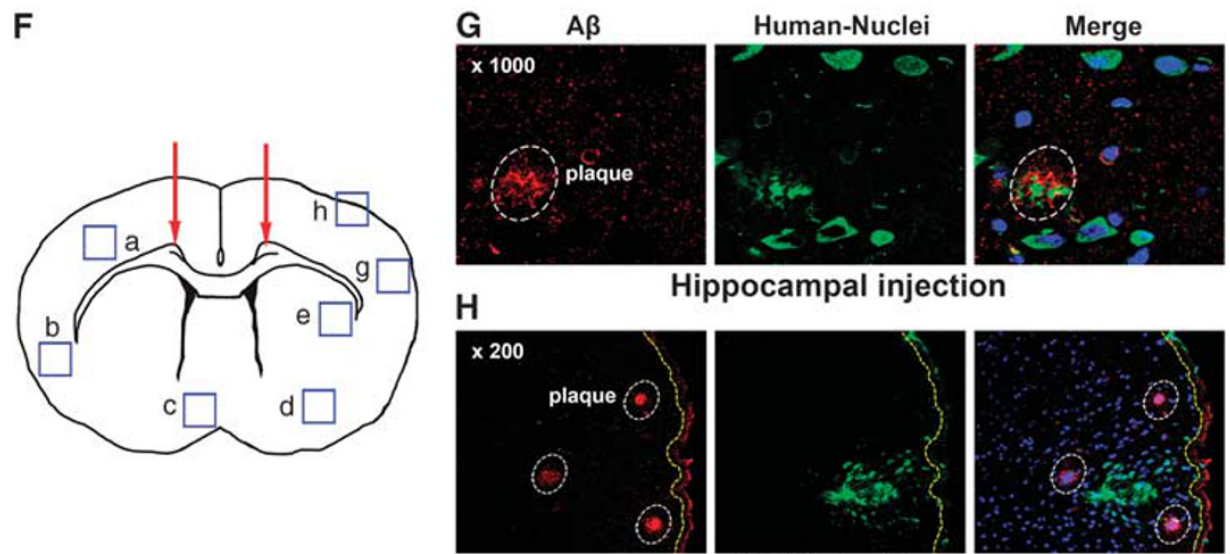

Hippocampal injection
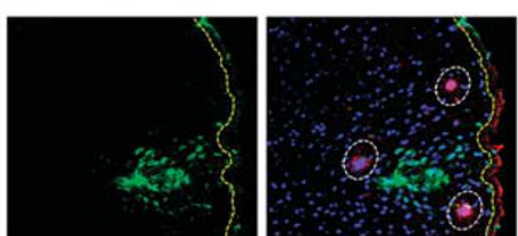

\section{CSF injection}

Figure 8 Active migration of hUCB-MSCs expressing ICAM-1 in mice brain. In the hUCB-MSC-transplanted groups (20-day), the frontal lobe area of brain tissues was co-stained by anti-human ICAM-1 (red), anti-human nuclei (green), and DAPI. (A-E) Regions from the neocortex, hypothalamus, amygdale, and striatum were observed by confocal microscopy. (F) In the coronal section, each box indicates regions where migrated hUCB-MSCs were detected by using each antibody. (G) Region $g$ in panel $\mathbf{F}, A \beta$ deposits were stained by using an anti-amyloid antibody (red). To detect migrated hUCB-MSCs near A $\beta$ deposits, hUCM-MSCs were stained by using an anti-human nuclei antibody. The white circles indicate A $\beta$ deposits in the brain. $(\mathrm{H})$ hUCB-MSCs were transplanted through the cisterna magna for CSF circulation. After 20 days, the cortex tissue under the subarchoid space (yellow dashed line) was stained by using a human nuclei and $A \beta$ antibody

amyloid- $\beta$ in vitro. ${ }^{30}$ By contrast, interruption of CD40/CD40L rescues $\mathrm{A} \beta$ deposition and tau hyper-phosphorylation in an AD animal model. ${ }^{31}$ Based on these reports, we can conclude that reduction of CD40 expression by hUCB-MNC administration must be involved in a therapeutic effect in the $A D$ model. As reported previously, CD40 expression was presently also downregulated by hUCB-MSC transplantation, compared with the control groups (Supplementary Figure S5a). Interestingly, we presently observed that exposure to recombinant ICAM-1 reduced CD40 expression in BV2 cells in 


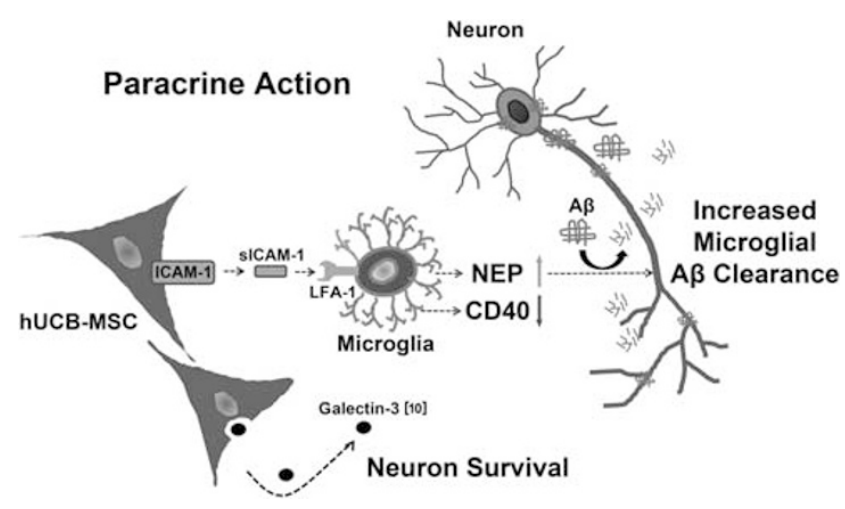

Figure 9 Therapeutic potential of hUCB-MSCs in AD. When hUCB-MSCs meet microglia, hUCB-MSCs secrete high levels of SICAM-1, which induces NEP expression, a key A $\beta$-degrading enzyme in microglia. Interestingly, hUCB-MSCderived sICAM-1 interrupted CD40/CD40L interaction on microglia through down regulation of CD40 expression in microglia. Recently, we reported that galectin-3 that is secreted by hUCB-MSCs protects against A $\beta 42$ neurotoxicity. ${ }^{10}$ Collectively, hUCB-MSCs may participate simultaneously in $A \beta$ clearance and neuronal survival through a paracrine mechanism in the $A D$ microenvironment

a time-dependent manner (Supplementary Figure S5b). From this result, we propose that sICAM-1 may also be a soluble factor to modulate CD40/CD40L activity.

As CD45, a tyrosine phosphatase (PTP), is a negative regulator of CD40L-induced microglial activation. ${ }^{32,33}$ To determine CD45 expression in hUCB-MSC injected groups, we also examined the expression of CD45 by western blot analysis. Although there is no significant difference in CD45 expression level between groups, we found a trend toward slight increase (by 20\%) in hUCB-MSC-transplanted mice as compared with controls (Supplementary Figure S5a). However, BV2 cells exposed to recombinant ICAM-1 did not show change of CD45 expression level (Supplementary Figure $\mathrm{S} 5 \mathrm{~b})$. Collectively, the data are consistent with the suggestion that hUCB-MSC-derived SICAM-1 can improve microglia function for $A \beta$ clearance through recovery of NEP expression and disruption of CD40/CD40L activity.

Although hUCB-MSC was injected into only the hippocampus, $A \beta$ plaques in other areas such as the remote cortex were also reduced (Figure 7). In our previous reports, hUCB-MSC migration toward IL-8 was too strong, because the injected hUCB-MSCs migrated toward the opposite hemispheres in the glioma region of nude mice at 7 days. Moreover, overexpression of the IL-8 receptor CXC chemokine receptor-1 (CXCR1) on hUCB-MSCs enhances cell migration in human glioma-bearing nude mice. ${ }^{19}$ Detection of hUCBMSCs expressing ICAM- 1 in other areas of the brain could be due to active migration of hUCB-MSCs to $A \beta$-exposed microglia secreting inflammatory cytokines (Figure 8).

Based on our data, we received clearance last year from the Korea Food and Drug Administration (KFDA) for a phase-I clinical trial of $A D$ (ClinicalTrials.gov Identifier: NCT01297218). We are now performing trials of administration of hUCB-MSCs in the brain parenchyma of AD patients. We suggest that, after considering relevant findings in total, hUCB-MSCs will constitute a new source of stem cell therapy against $A \beta$ plaques in clinical trials.

\begin{abstract}
Materials and Methods
Preparation of hUCB-MSCs and BV2 cells. This study was approved by the Institutional Review Board of MEDIPOST Co. Ltd. Umbilical cord blood was collected from umbilical veins after neonatal delivery, with informed consent from pregnant mothers. hUCB-MSCs were isolated and expanded according to the procedure specified in a previous report. ${ }^{10}$ To isolate and expand MSCs from cord blood, MNCs were removed by using a Ficoll-Hypaque solution $\left(d=1.077 \mathrm{~g} / \mathrm{cm}^{3}\right.$; Sigma-Aldrich Co., St Louis, MO, USA) and cells were then seeded at $5 \times 10^{5}$ cells/ $\mathrm{cm}^{2}$ in culture flasks. After colonies of spindle-shaped cells formed, the cells were reseeded for expansion. Hs68 cells (CRL-1635; ATCC, Rockville, MD, USA), which were used as control, were cultured under identical culture conditions as the hUCBMSCs. hUCB-MSCs $\left(8 \times 10^{4} \mathrm{cells} / \mathrm{cm}^{2}\right)$ were co-cultured in the upper chamber of a Transwell device (BD Biosciences, Franklin Lakes, NJ, USA) (pore size, $1 \mu \mathrm{m}$ ) with A $\beta 42$-exposed rat primary neuronal cells or BV2 cells. BV2 mouse microglia cells were provided by Professor Inhee-Mook Jung (Seoul National University).
\end{abstract}

Rat primary neuronal cultures. All animal procedures were conducted in accordance with institutional guidelines according to approved protocols. Pregnant Sprague-Dawley rats were purchased from OrientBio Inc. (Kyeonggi, Korea). Brain tissue was dissected from embryonic day 14 rat cortex and hippocampus, and cells were mechanically dissociated in $\mathrm{Ca}^{2+} / \mathrm{Mg}^{2+}$-free Hank's balanced salt solution. Cells were allowed to proliferate in the presence of $20 \mathrm{ng} / \mathrm{ml}$ basic fibroblast growth factor (bFGF) in serum-free neurobasal media. Cell proliferation was maintained in bFGF for 4 days to reach $80 \%$ confluence prior to induction of differentiation by withdrawal of the mitogen. Rat primary neuronal cultures were treated with $A \beta 42$ (Sigma-Aldrich) for 12 or $24 \mathrm{~h}$. To induce neurotoxicity, $A \beta 42$ aggregation was performed as described previously. ${ }^{34}$ Briefly, for the $A \beta 42$ oligomer, peptide was first resuspended in dimethylsulfoxide $\left(\mathrm{Me}_{2} \mathrm{SO}\right)$ to $5 \mathrm{mM}$ and then in cultured media to achieve a final peptide concentration of $100 \mu \mathrm{M}$. Peptide suspensions were incubated at $4{ }^{\circ} \mathrm{C}$ for $24 \mathrm{~h}$.

Western blot and ELISA. Cell extracts and tissues were prepared by ultrasonication (Branson Ultrasonics Corporation, Danbury, CT, USA) in buffer containing $9.8 \mathrm{M}$ urea, $4 \%$ CHAPS, $130 \mathrm{mM}$ dithiothreitol, $40 \mathrm{mM}$ Tris- $\mathrm{HCl}$, and $0.1 \%$ sodium dodecyl sulfate (SDS). Protein amount was measured by Bradford assay (Bio-Rad Laboratories, Hercules, CA, USA). Protein extract $(20 \mu \mathrm{g})$ was separated by SDS-PAGE and the resolved proteins were transferred to nitrocellulose membranes. In order to detect $A \beta 42$ in brain extracts, NuPAGE $12 \%$ Bis-Tris gels (Invitrogen, Carlsbad, CA, USA) were used to separate lowmolecular-weight proteins by SDS-PAGE. Each membrane was incubated with antiNEP (R\&D Systems, Minneapolis, MN, USA), anti-IDE (Calbiochem, San Diego, CA, USA), and anti-A $\beta 42$ (Novus Biologicals, LLC, Littleton, CO, USA). ELISA was performed according to the manufacturer's instructions. An ICAM-1 ELISA kit (R\&D Systems) and kits for $A \beta 40$ or $A \beta 42$ (Wako Pure Chemical Industries Ltd, Osaka, Japan) were used. For analysis of soluble and insoluble $A \beta 40$ and 42 , brain tissues were sonicated in ice-cold PBS containing 1\% Triton X-100, $20 \mathrm{mM}$ Tris, $150 \mathrm{mM}$ $\mathrm{NaCl}, 1 \mathrm{mM} E D T A$, and a protease/phosphatase inhibitor cocktail. After centrifugation at 13000 r.p.m. for $15 \mathrm{~min}$ at $4{ }^{\circ} \mathrm{C}$, the supernatant was analyzed as a soluble fraction and the pellet was resolubilized in $5 \mathrm{M}$ guanidine buffer. ${ }^{29}$

IHC and thioflavin-S staining. For IHC of mouse brain, mice were killed after hUCB-MSC transplantation. After transplantation, the animals immediately underwent cardiac perfusion with $4 \%$ paraformaldehyde in PBS. After perfusion, post-fixation was performed overnight at $4{ }^{\circ} \mathrm{C}$, and the brains were then incubated again in $30 \%$ sucrose at $4{ }^{\circ} \mathrm{C}$ until they were equilibrated. Sequential $16-\mu \mathrm{m}$ coronal cryosections were obtained on a CM1850UV cryostat microtome (Leica Microsystems Inc., Jena, Germany) and stored at $-20^{\circ} \mathrm{C}$. Tissues or cells were stained with antibodies for MAP2 (Millipore, Billerica, MA, USA), tubulin- $\beta$ III (Sigma-Aldrich), human nuclei (Millipore), human $\beta 2$-microglobulin, A $\beta$ (Abcam, Cambridge, MA, USA), or CD11b (AbD Serotec, Kidlington, UK). Fluorescein isothiocyanate (FITC, Cy3)-labeled secondary antibodies (Jackson Immunoresearch Laboratories, Bar Harbor, MN, USA) or the VECTASTAIN Elite ABC Kit (Vector Laboratories, Burlingame, CA, USA) were used and photographed using a confocal microscope (Nikon Corporation, Tokyo, Japan). For thioflavin-S staining, tissues were placed in $1 \%$ thioflavin-S solution (Sigma-Aldrich) and then differentiated in $70 \%$ ethanol. $A \beta$ plaques were examined by quantitative image analysis using the Metamorph 7.1.2 software (Molecular Devices, Sunnyvale, CA, USA). 
siRNA treatment. siRNAs for NEP, LFA-1, and ICAM-1 were purchased from Bioneer Corporation (Daejeon, Korea). BV2 cells and hUCB-MSCs were treated with NEP siRNA and ICAM-1 siRNA, respectively, for 8-14h using the Lipofectamine 2000 reagent (Invitrogen). These cells were cultured in complete media overnight and co-cultured with hUCB-MSCs or BV2 cells.

Administration of hUCB-MSCs in mice. Double transgenic mice, $\mathrm{Mo} / \mathrm{Hu}$ APPswe PS1dE9: APP/PS1 were purchased from Jackson Laboratory (Bar Harbor, ME, USA) and offsprig mice were produced, selected and maintained according to the protocols recommended by Jackson Laboratory. Ten-month-old APP/PS1 mice or their $\mathrm{B} 6 \mathrm{C} 3$ littermates were anesthetized with ketamine/xylazine intraperitoneally and fixed in a stereotaxic apparatus (Stoelting, Wood Dale, IL, USA) for hUCB-MSC transplantation. Bilateral injections were performed by injecting $3 \mu \mathrm{l}$ of the cell suspension ( $1 \times 10^{4}$ cells per hippocampus) or recombinant ICAM-1 protein into the dentate gyrus of the hippocampus, or $15 \mu$ l of the same number of cells into cisterna magna. hUCB-MSCs were stereotactically inoculated into the hippocampal area (AP: $-2.3, \mathrm{~L}: \pm 1.3$, DV: -2.0 referenced to the bregma) or the cisterna magna with a sterile Hamilton syringe fitted with a 26-gauge needle (Hamilton Company, Reno, NV, USA), using a microinfusion pump (KD Scientific, Holliston, MA, USA) in a stereotaxic apparatus. The cell suspension was delivered at a rate of $0.5 \mu \mathrm{l} / \mathrm{min}$.

\section{Conflict of Interest}

The authors declare no conflict of interest.

Acknowledgements. This study was supported by a grant of the Korea Healthcare technology R\&D Project, Ministry of Health \& Welfare, Republic of Korea (A110445)

1. Querfurth HW, LaFerla FM. Alzheimer's disease. N Engl J Med 2010; 362: 329-344.

2. Miller G. Pharmacology. The puzzling rise and fall of a dark-horse Alzheimer's drug. Science 2010; 327: 1309.

3. Salem HK, Thiemermann C. Mesenchymal stromal cells: current understanding and clinical status. Stem Cells 2010; 28: 585-596.

4. Secco M, Zucconi E, Vieira NM, Fogaca LL, Cerqueira A, Carvalho MD et al. Multipotent stem cells from umbilical cord: cord is richer than blood!. Stem Cells 2008; 26 $146-150$

5. Oh W, Kim DS, Yang YS, Lee JK. Immunological properties of umbilical cord blood-derived mesenchymal stromal cells. Cell Immunol 2008; 251: 116-123.

6. Sanberg PR, Eve DJ, Willing AE, Garbuzova-Davis S, Tan J, Sanberg CD et al. The treatment of neurodegenerative disorders using umbilical cord blood and menstrual bloodderived stem cells. Cell Transplant 2011; 20: 85-94.

7. Block GJ, Ohkouchi S, Fung F, Frenkel J, Gregory C, Pochampally R et al. Multipotent stromal cells are activated to reduce apoptosis in part by upregulation and secretion of stanniocalcin-1. Stem Cells 2009; 27: 670-681.

8. Lee RH, Pulin AA, Seo MJ, Kota DJ, Ylostalo J, Larson BL et al. Intravenous hMSCs improve myocardial infarction in mice because cells embolized in lung are activated to secrete the anti-inflammatory protein TSG-6. Cell Stem Cell 2009; 5: 54-63.

9. Karaoz E, Genc ZS, Demircan PC, Aksoy A, Duruksu G. Protection of rat pancreatic islet function and viability by coculture with rat bone marrow-derived mesenchymal stem cells. Cell Death Dis 2010; 1: e36.

10. Kim JY, Kim DH, Kim DS, Kim JH, Jeong SY, Jeon HB et al. Galectin-3 secreted by human umbilical cord blood-derived mesenchymal stem cells reduces amyloid-beta42 neurotoxicity in vitro. FEBS Lett 2010; 584: 3601-3608.

11. Lee HJ, Lee JK, Lee H, Shin JW, Carter JE, Sakamoto T et al. The therapeutic potential of human umbilical cord blood-derived mesenchymal stem cells in Alzheimer's disease. Neurosci Lett 2010; 481: 30-35

12. Lee HJ, Lee JK, Lee H, Carter JE, Chang JW, Oh W et al. Human umbilical cord bloodderived mesenchymal stem cells improve neuropathology and cognitive impairment in an Alzheimer's disease mouse model through modulation of neuroinflammation. Neurobio Aging 2010; e-pub ahead of print 13 May 2010.
13. Iwata N, Tsubuki S, Takaki Y, Watanabe K, Sekiguchi M, Hosoki E et al. Identification of the major Abeta1-42-degrading catabolic pathway in brain parenchyma: suppression leads to biochemical and pathological deposition. Nat Med 2000; 6: 143-150.

14. Sigal A, Bleijs DA, Grabovsky V, van Vliet SJ, Dwir O, Figdor CG et al. The LFA-1 integrin supports rolling adhesions on ICAM-1 under physiological shear flow in a permissive cellular environment. J Immunol 2000; 165: 442-452.

15. Roescher N, Vosters JL, Yin H, Illei GG, Tak PP, Chiorini JA. Effect of soluble ICAM-1 on a Sjogren's syndrome-like phenotype in NOD mice is disease stage dependent. PLOS One 2011; 6: e19962.

16. Vekrellis K, Ye Z, Qiu WQ, Walsh D, Hartley D, Chesneau V et al. Neurons regulate extracellular levels of amyloid beta-protein via proteolysis by insulin-degrading enzyme. Neurosci 2000; 20: 1657-1665.

17. Kim DS, Kim JH, Lee JK, Choi SJ, Kim JS, Jeun SS et al. Overexpression of CXC chemokine receptors is required for the superior glioma-tracking property of umbilical cord blood-derived mesenchymal stem cells. Stem Cells Dev 2009; 18: 511-519.

18. Kim SM, Oh JH, Park SA, Ryu CH, Lim JY, Kim DS et al. Irradiation enhances the tumor tropism and therapeutic potential of tumor necrosis factor-related apoptosis-inducing ligand-secreting human umbilical cord blood-derived mesenchymal stem cells in glioma therapy. Stem Cells 2010; 28: 2217-2228.

19. Kim SM, Kim DS, Jeong $\mathrm{CH}$, Kim DH, Kim JH, Jeon HB et al. CXC chemokine receptor 1 enhances the ability of human umbilical cord blood-derived mesenchymal stem cells to migrate toward gliomas. Biochem Biophys Res Commun 2011; 407: 741-746.

20. Dickson DW. Microglia in Alzheimer's disease and transgenic models. How close the fit? Am J Pathol 1999; 154: 1627-1631.

21. El Khoury J, Hickman SE, Thomas CA, Loike JD, Silverstein SC. Microglia, scavenge receptors, and the pathogenesis of Alzheimer's disease. Neurobiol Aging 1998; 19 (1 Suppl): S81-S84

22. Walker DG, Lue LF, Beach TG. Gene expression profiling of amyloid beta peptide-stimulated human post-mortem brain microglia. Neurobiol Aging 2001; 22: 957-966.

23. Blurton-Jones M, Kitazawa M, Martinez-Coria $\mathrm{H}$, Castello NA, Muller FJ, Loring JF et al. Neural stem cells improve cognition via BDNF in a transgenic model of Alzheimer disease. Proc Natl Acad Sci USA 2009; 106: 13594-13599.

24. Wu S, Sasaki A, Yoshimoto R, Kawahara Y, Manabe T, Kataoka K et al. Neural stem cells improve learning and memory in rats with Alzheimer's disease. Pathobiology 2008; 75 : 186-194

25. Xuan AG, Luo M, Ji WD, Long DH. Effects of engrafted neural stem cells in Alzheimer's disease rats. Neurosci Lett 2009; 450: 167-171.

26. Mawuenyega KG, Sigurdson W, Ovod V, Munsell L, Kasten T, Morris JC et al. Decreased clearance of CNS beta-amyloid in Alzheimer's disease. Science 2010; 330: 1774.

27. Rieckmann P, Michel U, Albrecht M, Bruck W, Wockel L, Felgenhauer K. Soluble forms of intercellular adhesion molecule-1 (ICAM-1) block lymphocyte attachment to cerebral endothelial cells. J Neuroimmunol 1995; 60: 9-15

28. Li D, Molldrem JJ, Ma Q. LFA-1 regulates CD8+ $T$ cell activation via $T$ cell receptor-mediated and LFA-1-mediated Erk1/2 signal pathways. J Biol Chem 2009; 284: 21001-21010.

29. Nikolic WV, Hou H, Town T, Zhu Y, Giunta B, Sanberg CD et al. Peripherally administered human umbilical cord blood cells reduce parenchymal and vascular beta-amyloid deposits in Alzheimer mice. Stem Cells Dev 2008; 17: 423-439.

30. Townsend KP, Town T, Mori T, Lue LF, Shytle D, Sanberg PR et al. CD40 signaling regulates innate and adaptive activation of microglia in response to amyloid beta-peptide. Eur J Immunol 2005; 35: 901-910.

31. Tan J, Town T, Crawford F, Mori T, DelleDonne A, Crescentini R et al. Role of CD40 ligand in amyloidosis in transgenic Alzheimer's mice. Nat Neurosci 2002; 5: 1288-1293.

32. Tan J, Town T, Mullan M. CD45 inhibits CD40L-induced microglial activation via negative regulation of the Src/p44/42 MAPK pathway. J Biol Chem 2000; 275: 37224-37231.

33. Zhu Y, Hou H, Rezai-Zadeh K, Giunta B, Ruscin A, Gemma C et al. CD45 deficiency drives amyloid-beta peptide oligomers and neuronal loss in Alzheimer's disease mice. J Neurosci 2011; 31: 1355-1365.

34. Dahlgren KN, Manelli AM, Stine Jr WB, Baker LK, Krafft GA, LaDu MJ. Oligomeric and fibrillar species of amyloid-beta peptides differentially affect neuronal viability. J Biol Chem 2002: 277: 32046-32053.

This work is licensed under the Creative Commons Attribution-NonCommercial-No Derivative Works 3.0 Unported License. To view a copy of this license, visit http:/l creativecommons.org/licenses/by-nc-nd/3.0

Supplementary Information accompanies the paper on Cell Death and Differentiation website (http://www.nature.com/cdd) 